Article

\title{
Acrylic Pressure-Sensitive Adhesive Reinforced with Aluminum Nitride and Its Thermal Properties: Effect of Surface Treatment and Particle Size
}

\author{
Garima Mittal ${ }^{1}$, Soo Jin Park ${ }^{2, *}$ and Kyong Yop Rhee ${ }^{1, * \mathbb{C}}$ \\ 1 Department of Mechanical Engineering, College of Engineering, Kyung Hee University, Yongin 17104, Korea; \\ garima.nano@gmail.com \\ 2 Department of Chemistry, Inha University, 100 Inharo, Incheon 22212, Korea \\ * Correspondence: sjpark@inha.ac.kr (S.J.P.); rheeky@khu.ac.kr (K.Y.R.); Tel.: +82-32-8767234 (S.J.P.); \\ +82-31-2012565 (K.Y.R.); Fax: +82-32-863-1333 (S.J.P.); +82-31-2026693 (K.Y.R.)
}

Received: 24 December 2019; Accepted: 18 February 2020; Published: 22 February 2020

\begin{abstract}
Thermal interface materials (TIMs) are very crucial for better heat-transfer in electronics working as an interfacial connection between heat generators and heat sinks. This study is focused on the pressure-sensitive acrylic adhesive tape reinforced with micron-sized and nano-sized aluminum nitride (AlN) particles where the surface modification of AlN particles is done using (3-Aminopropyl) triethoxysilane (3-APTES). The physicochemical analysis of the silanized AlN particles is done using FTIR spectroscopy and scanning electron microscopy (SEM). Furthermore, thermal properties along with thermal conductivity and thermal diffusion are also studied. The main outcome of this study shows that the sample containing surface-treated AlN particles exhibits better thermal conductivity than that of the samples containing $\mu$ and nano-sized of AlN due to the comparatively better interactions with the matrix.
\end{abstract}

Keywords: pressure sensitive adhesive; aluminum nitride; surface treatment; thermal conductivity

\section{Introduction}

In modern electronic devices such as notebook PCs, electric cars, and LEDs, thermal management is a crucial issue. With the miniaturization of these modern devices, heat generation and its dissipation have become a challenging problem. Improper heat dissipation can cost the performance, reliability, and lifetime of the device [1]. Hence, thermal interface materials (TIMs) are very crucial for better heat-transfer in electronics working as an interfacial connection between heat generators and heat sinks. There are different types of TIMs used on commercial bases such as thermal grease, thermal gap pads, phase change materials, and form-in-place compounds. However, due to drawbacks like the requirement of pressure or mechanical clamping, contamination, degradation, and less adhesion, these types of TIMs are not favored commercially [2]. Contrarily, thermally conductive adhesive overcome all the problems and assure an exceptional bonding property between electrical components and heat sink, which eliminates the need for external clamping. Silicone, acryl, or epoxy-based double-sided thermal tapes are commonly used as thermally conductive adhesives. These tapes are thermally, mechanically, and environmentally more sustainable, more suitable for a variety of applications, and easy to design [3]. Along with the outstanding thermal conductivity, good adhesive strength and re-workability are desired in an ideal thermal conductive tape. Commercially, these tapes are used to attach heat sinks to PC processors, motor control processors, or telecommunication infrastructure components [4].

Acryl-based pressure sensitive adhesives (PSAs) are in demand from the industrial point of view due to their low-cost, durability, and secure processing. The resistivity toward the temperature, 
chemicals, sunlight, and other radiations make it an approachable PSA for thermal applications [5]. The performance of these acryl-based adhesive tapes excessively depends on the monomer, cross-linkers, and initiators [6]. To further increase the performance of these acrylic PSA tapes, thermally conductive fillers are incorporated into the polymer matrix such as metal particles, graphene, carbon nanotubes (CNTs), graphite, hexagonal boron nitride (hBN), aluminum nitride (AlN), and aluminum oxide [7]. In some applications, high thermal conductivity, along with an electrically insulating property, is required to avoid the danger of short circuits. In those cases, metal oxides are used as fillers that lack free electrons and transfer the heat through phonons. AlN and hBN are highly electrically insulating with the highest thermal conductivity among all the ceramic fillers. However, the exceptionally low thermal conductivity in the through-plane direction makes it difficult to use hBN as a filler [8]. On the other hand, surface treatment of AlN particles prior to the incorporation into the polymer matrix is required to avoid the adverse effect of the moisture on the particles.

The surface treatment is considered as an effective tool to disperse the material into the polymer matrix. It can be practiced via various methods such as chemical functionalization, cryo-milling, thermal treatment, and plasma treatment [9]. When particles are mixed into the matrix, numerous interfaces are created, which limits the thermal conductivity of the final composite by increasing the interfacial resistance (thermal contact resistance and thermal boundary resistance) [10]. Towards improving the thermal contact resistance, filler and polymers are bonded together using the surface modifiers permanently attached to the surface of filler so that the chemical groups of polymers can make a connection with them [11]. Hence, surface treatment improves the interfacial interaction and adhesion between the filler and polymer. Along with surface properties, filler type, filler size, filler loading, filler shape, and filler orientation also affect the final performance of the composite [12]. For instance, even though the aspect is still controversial, nanosized fillers enhance the thermal conductance by forming an interconnected network that decreases the phonon scattering. Whereas, in contrast to the nanosized fillers and based on a different mechanism, micro-sized fillers in a composite exhibit lesser filler/matrix interface, which lowers the phonon scattering and leads to better thermal conduction. These two examples, according to some reported data [12], could be rationally explained by the fact that the interfacial resistance is more critical than the interconnection [12].

As per our knowledge, given the relatively small number of research reports available on acrylic pressure-sensitive adhesives, and, since the thermal performance of the composite material depends on the structure and properties of the polymer as well, the results reported herein could be useful from an applicative point of view. In this study, we are synthesizing an AlN reinforced acrylic pressure-sensitive adhesive, where AlN is mixed into the acryl matrix in different sizes (nano and micro), and the surface of micro-AlN is treated with a silane coupling agent. To compare the effects of surface treatment and particle size on the thermal conductivity of the final systems, we have prepared different polymer-filler compositions. The physicochemical study of the AlN particles before and after the surface treatment is performed using FTIR spectroscopy and SEM. Thermal properties of composites are analyzed by TGA, DSC, thermal conductivity, thermal diffusion, and specific heat measurements.

\section{Materials and Methods}

\subsection{Materials Required}

The UV-curable hydroxyalkyl acrylate-based monomer, which was a combination of 2-hydroxyethyl acrylate and 2-hydroxypropyl acrylate and a polymerizing agent were purchased from ISA CO. LTD., Korea. Aluminum nitride particles $(10 \mu \mathrm{m}$ and $10 \mathrm{~nm})$ purchased from Sigma Aldrich, Seoul, Korea were used as a thermally conductive filler material. To treat the surface of AlN particles, 3-Aminopropyl triethoxysilane (3-APTES) was used and was purchased from Sigma Aldrich. The distilled water and ethanol were obtained from Daejung chemicals, Siheung-si, Korea. The UV lamp used for curing the PSA/filler mixture was a linear Type UV-LED Curing System. 


\subsection{Surface Treatment of AlN Particles}

The surface of AlN particles was modified by the silanization method. Before silanization, the surface of AlN particles was cleaned by sonicating the particles in ethanol for $1 \mathrm{~h}$, which was followed by filtering and drying at room temperature. For silanization, $5 \mathrm{wt} . \%$ of 3-APTES, based on the total weight of the treated AlN particles was added to a mixed solution of $190 \mathrm{~mL}$ of ethanol and $10 \mathrm{~mL}$ of water and stirred for $15 \mathrm{~min}$. Furthermore, the desired amount of sonicated AlN particles was mixed into the above mixture and stirred for $6 \mathrm{~h}$ at a constant temperature of $70{ }^{\circ} \mathrm{C}$. Afterward, the treated particles were filtered and washed many times, and followed by drying in an oven at $80^{\circ} \mathrm{C}$. These treated AlN particles are referred to as Si-AlN.

\subsection{Synthesis of PSA/AlN Composites}

PSA/AlN composites were prepared by mechanically mixing the AlN particles with the mixture of the UV sensitive PSA resin and hardener, which was cured by baking under the presence of UV light (Figure 1). For a better dispersion of AlN particles into the matrix, a combination of mechanical mixing and sonication was used. The heat generated through sonication made the resin comparatively less viscous and aided in better dispersion of AlN particles into the matrix. In addition, the bubbles were removed from the matrix using sonication that was beneficial as well. Furthermore, the reinforced resin was spread over the silicon-coated side of a transparent PET film with the help of a knife coater (Model: KP-3000 V, Suwon, Korea, Speed: $5 \mathrm{~mm} / \mathrm{sec}$ ) and cover with the silicon-coated side of a transparent PET film. Later, the sandwiched system with the resin in the middle was cured under a UV light for several minutes. Usually, in order to make a proper thermally conductive network, more than $30 \mathrm{wt}$ \% of thermally conductive filler is used [13]. In addition, in some cases, the use of different sizes ( $\mu$ and nano) of filler materials has been reported [14]. In this study, three combinations of composites were prepared that are referred to as $\mu$-AlN/n-AIN/PSA, Si- $\mu$-AlN/n-AlN/PSA, and Si- $\mu$-AlN/PSA (Table 1). Filler-free PSA samples were also prepared as a reference.

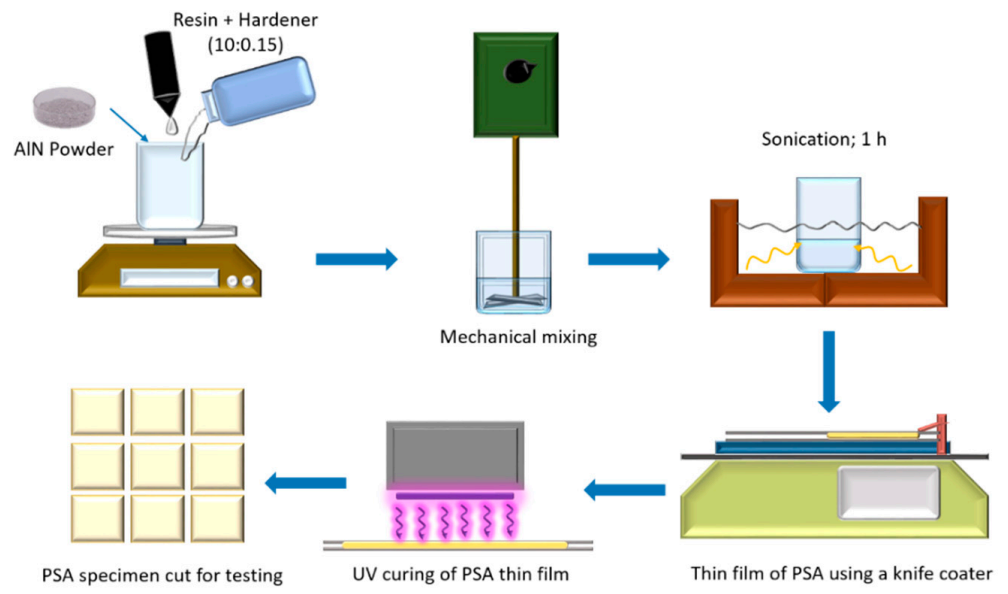

Figure 1. Schematic representation of the synthesis of acrylic pressure-sensitive/ aluminum nitride (AlN) composites.

Table 1. Composition of different acrylic pressure-sensitive composite samples.

\begin{tabular}{cccccc}
\hline \multirow{2}{*}{ No. } & \multirow{2}{*}{ Sample Name } & \multirow{2}{*}{ Resin (g) } & Curing Agent (g) & \multicolumn{2}{c}{ Filler (g) } \\
\cline { 5 - 6 } & & 20 & & $\mu .3$ & $\mathbf{n}$ \\
\hline 1. & PSA & 20 & 0.3 & 27 & - \\
2. & $\mu$-AlN/n-AlN/PSA & 20 & 0.3 & 37 & 3 \\
3. & Si- $\mu$-AlN/n-AlN/PSA & 20 & 0.3 & 40 & - \\
4. & Si- $\mu$-AlN/PSA & & & & \\
\hline
\end{tabular}




\subsection{Characterization}

Fourier transformed infrared (FTIR) spectroscopy using the Attenuated Total Reflectance (ATR) method was performed. The infrared (IR) spectra were collected in the wavenumber range of $400-4000 \mathrm{~cm}^{-1}$. The morphology of AlN particles before and after the surface modification was compared using field-emission scanning electron microscopy (FE-SEM, Carl Zeiss, Germany). The surface and cross-sectional morphologies of PSA/AlN composites were also examined. Before analysis, the samples were coated with $\mathrm{Pt}$ via sputtering for better conductivity. Thermal degradation patterns and the associated weight loss were studied using thermogravimetric analysis, which was performed in the presence of nitrogen gas $(10 \mathrm{~mL} / \mathrm{min})$ in the temperature range of 25 to $800^{\circ} \mathrm{C}$ at a heating rate of $10^{\circ} \mathrm{C} / \mathrm{min}$. The melting behavior of the composites was comparatively investigated using differential scanning calorimetry (DSC). On the other hand, thermal conductivity, thermal diffusivity, and specific heat were measured by employing a Hot Disk thermal constant analyzer (Hot Disk, Inc. USA) using a Kapton sensor tape meeting ISO Standard 22007-2 [15]. This technique is based upon the Transient Plane Source (TPS) method, where the probe is a spiral-shaped resistive element made of a metal foil covered with Kapton film from both sides acting as a heat source and sensor simultaneously. For analysis, this probe is sandwiched between two pieces of the test sample (same material). During heating, the spiral element via the electric current pulse, the increase in the resistance as a function of time is recorded to give thermal conductivity.

\section{Results}

\subsection{Physicochemical Properties}

To confirm the successful silane-treatment on the surface of AlN particles, FTIR spectroscopy (PerkinElmer, Waltham, MA, USA) was used. Figure 2 shows the comparative spectra of non-treated AlN and Si-AlN. Since the samples were analyzed using the ATR method, the peak related to the adsorbed moisture is absent. Non-treated AIN display a broad absorption peak ranging from a 950 to $500 \mathrm{~cm}^{-1}$ wavelength (a) as well as a sharp peak at $1330 \mathrm{~cm}^{-1}$ wavelength (b) ascribed to vibrations of the Al-N bond [16]. While, in the case of $\mathrm{Si}-\mathrm{AlN}$, some additional peaks were also observed such as a broad peak from 3100 to $3400 \mathrm{~cm}^{-1}$ attributed to the $-\mathrm{OH}$ and $-\mathrm{NH}_{2}$, two peaks positioned at 2916 and $2847 \mathrm{~cm}^{-1}$ due to the alkyl groups, a peak at $1648 \mathrm{~cm}^{-1}$ related to the $\mathrm{N}-\mathrm{H}$ bending belonging to $-\mathrm{NH}_{2}$ groups, and peaks at around 1181 and $1067 \mathrm{~cm}^{-1}$ assigned to the Si-O-R groups of APTES [17].

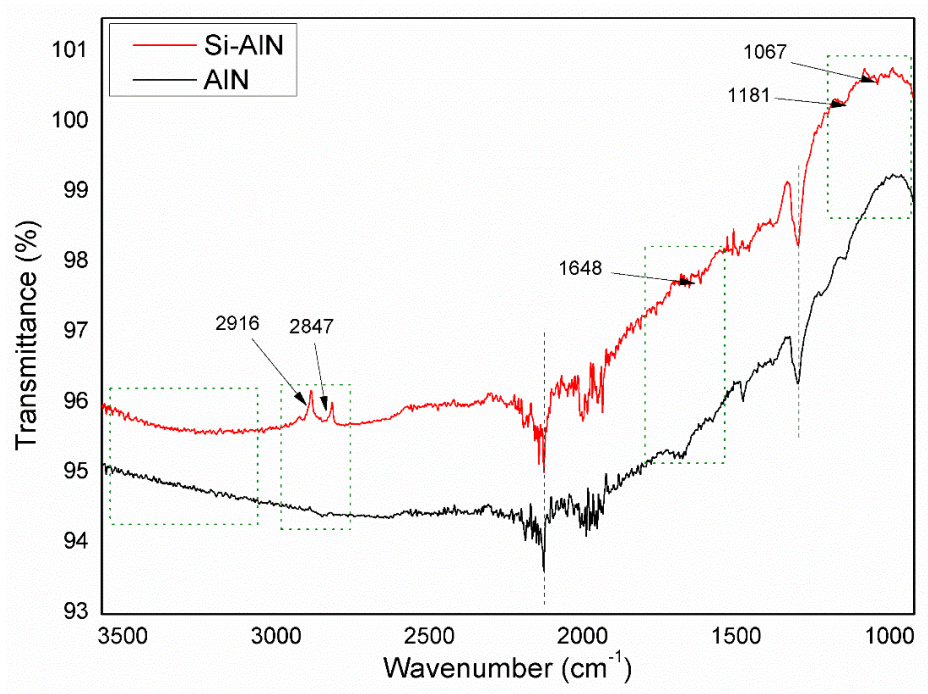

Figure 2. Fourier-transform infrared spectroscopy (FTIR) spectra of non-treated aluminum nitride (AlN) and Si-AlN. 
Figure 3 compares the FE-SEM images of the surface morphology of AlN particles before and after the silane treatment. As can be seen in Figure 3a, the surface of AlN particles was smoother with sharp cuts, which became rougher after silane treatment (Figure $3 b$ ). The reason behind this roughness is the attachment of chemical groups presented in the coupling agent that reacted with the -OH group at the surface of AlN [18].

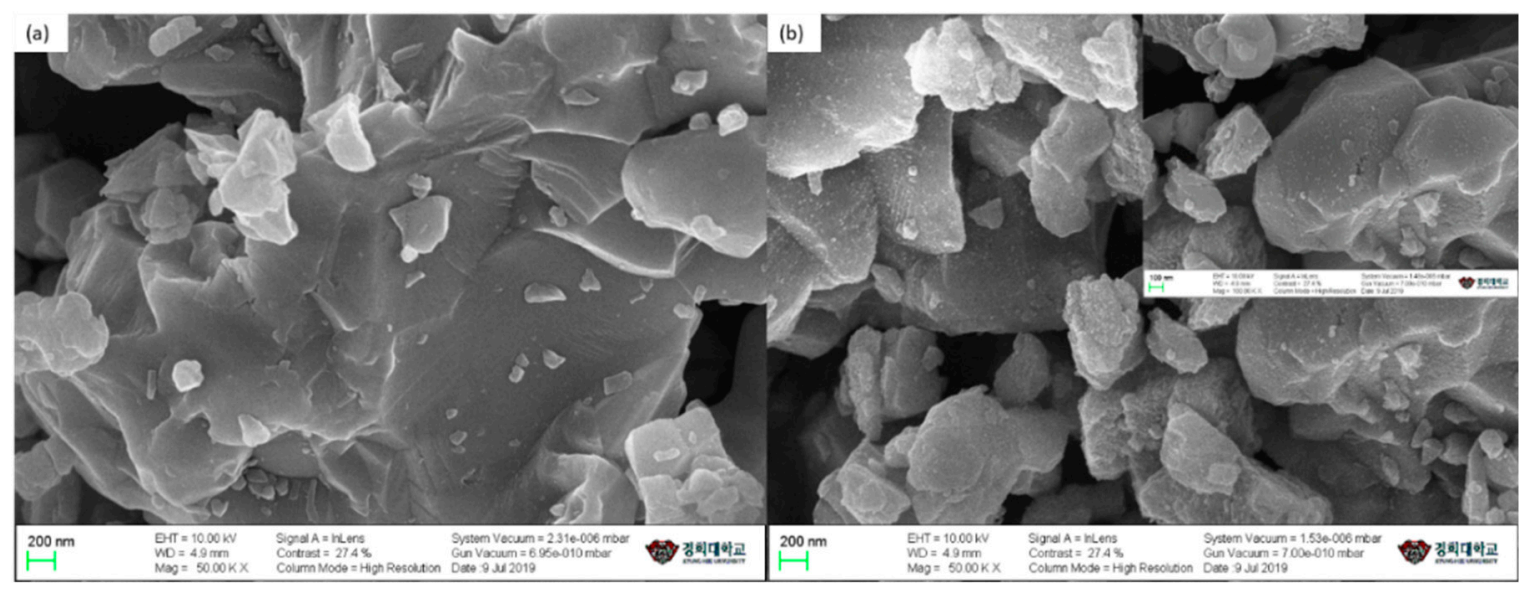

Figure 3. FE-SEM images of (a) non-treated AlN and (b) Si-AlN (Inset: $100 \mathrm{KX).}$

The surface morphology of different PSA composites was compared using FE-SEM and shown in Figure 4. Figure 4a-f represent the FE-SEM images of $\mu$-AlN/n-AlN/PSA, Si- $\mu-A 1 N / n-A 1 N / P S A$, and $\mathrm{Si}-\mu$-AlN/PSA, respectively. To improve the image quality, the surface of all the samples was coated with platinum. Since the neat PSA sample has a very low thermal stability, it was not possible to take FE-SEM images. The sample started melting as soon as the electron beam was focused on it. From images, it was found that the sample $\mu$-AlN/n-AIN/PSA shows the roughest surface as compared to the others, which indicates a great degree of agglomeration. This happened due to the lack of interactions between the non-treated particles and matrix, which was even confirmed in Figure $4 \mathrm{~b}$. Figure $4 \mathrm{~b}$ is an SEM image of the edge of the sample from where it was cut. Since bare AlN particles do not have any compatible chemical functional group that can interact with the acrylate group present in the matrix that leads to poor dispersion of AlN particles into the matrix. The surface of the $\mathrm{Si}-\mu-\mathrm{AlN} / \mathrm{n}$-AlN/PSA was comparatively smoother, which indicates a proper dispersion of $\mu$-AlN and $n$-AlN particles into the matrix. As can be seen from Figure $4 d$, the edge of the sample was smoother than the $\mu$-AlN/n-AlN/PSA because functional groups presented on the silane-treated $\mu$-AlN surface formed bonds with chemical groups of PSA improving the interactions between AIN particles and the matrix. During the silanization process, APTES molecules are chemosorbed onto the surface of AlN particles by forming chemical bonds of type "AlN-O-Si-" as a result of a reaction between the possible -OH groups on the AlN surface and the ethoxy moiety of APTES. In this case, it corresponds to the amounts of ethanol generated as a by-product. Afterward, the functionalized surface (with $-\mathrm{NH}_{2}$ groups) of AlN particles could strongly interact via hydrogen bonding with the PSA matrix (ester groups), which enhances the strength of adhesion between the inorganic (AlN particles) and organic (PSA matrix) phases of the mixed systems. Particles were more evenly dispersed in Si- $\mu$-AlN/PSA as compared to the $\mathrm{Si}-\mu-\mathrm{AlN} / \mathrm{n}-\mathrm{AlN} / \mathrm{PSA}$ because all the AlN were treated with the coupling agent that improved the dispersion and interactions between chemical groups present on the surface of AlN particles with chemical moieties of the matrix [19]. In addition, due to the absence of the non-treated n-AIN particles, a proper conducting network was formed. The FE-SEM image of the edge of the cut sample was in accordance with the SEM image of the surface. 

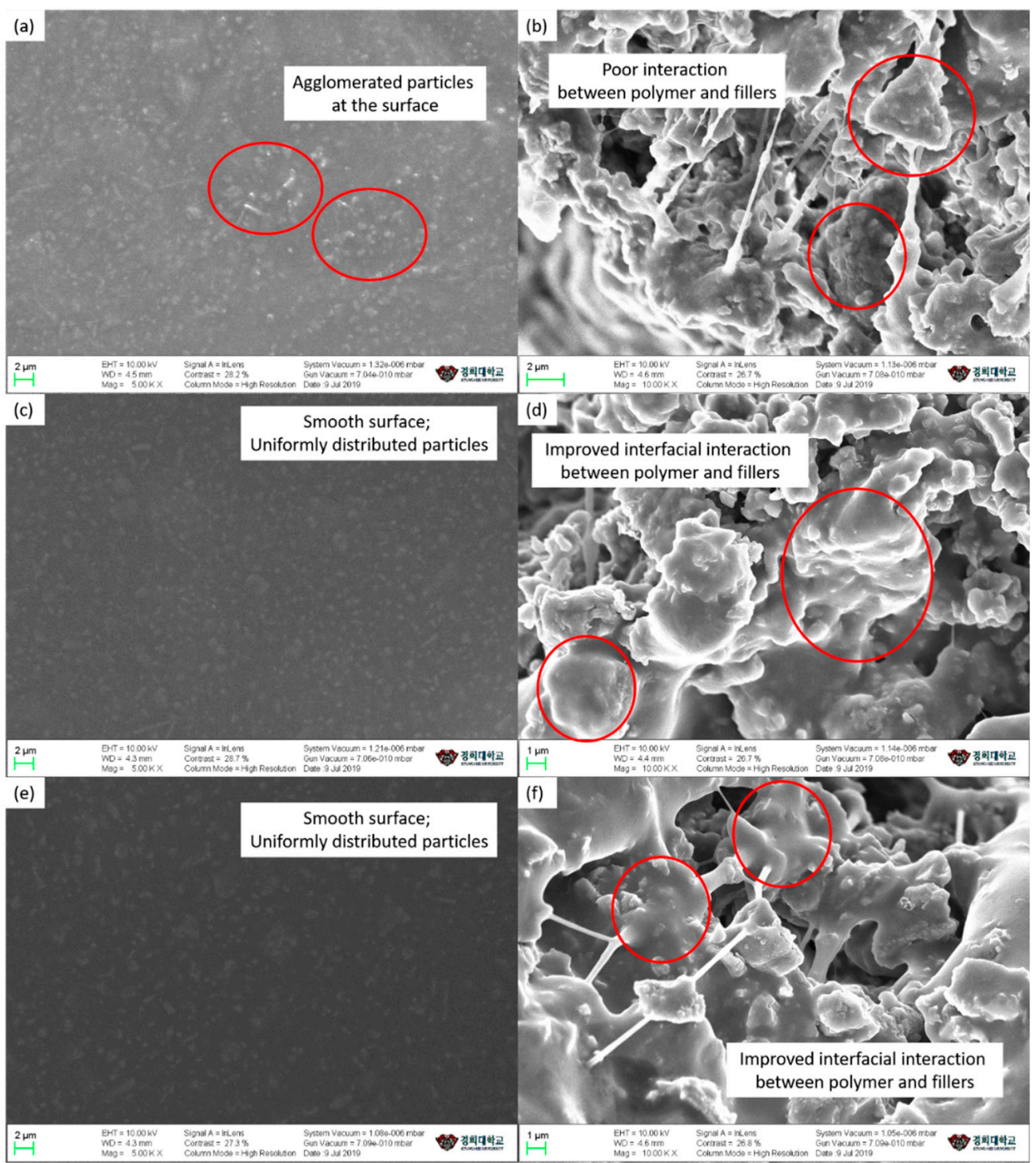

Figure 4. FE-SEM images of (a,b) $\mu$-AlN/n-AlN/PSA, (c,d) Si- $\mu$-AlN/n-AlN/PSA, and (e,f) Si- $\mu$-AlN/PSA, respectively, where (a,c, and $\mathbf{e})$ are showing surface morphology, while $(\mathbf{b}, \mathbf{d}$, and $\mathbf{f})$ are the cutting edge of samples.

\subsection{Thermal Properties}

To study the thermal behavior of the prepared samples, TGA was performed. Figure 5 displays the TGA patterns of PSA, $\mu$-AlN/n-AlN/PSA, Si- $\mu$-AlN/n-AlN/PSA, and Si- $\mu$-AlN/PSA, respectively. Of all the studied systems, neat PSA exhibits the highest weight loss that means the lowest thermal stability. The TGA pattern of neat PSA shows two-steps while other samples have a three-steps degradation pattern. These two steps of degradation in neat PSA are ascribed to the degradation of polymer chains (at around $290^{\circ} \mathrm{C}$ ) and total decomposition of the polymer (at around $370{ }^{\circ} \mathrm{C}$ ) in PSA, respectively [20]. In all the AlN reinforced PSA composites, one extra step at around $100^{\circ} \mathrm{C}$, ascribed to the adsorbed moisture on AlN particles due to their hygroscopic nature, is also observed. Table 2 indicates the observed weight loss in different steps for different samples. To compare the thermal stability, the heat-resistance index is also calculated using Equation (1) at the decomposition temperature for $5 \%$ and $30 \%$ weight loss $\left(T_{5}\right.$ and $\left.T_{30}\right)$, respectively [21].

$$
\text { Heat-resistance index }=0.49 \times\left[T_{5}+0.6 \times\left(T_{30}-T_{5}\right)\right]
$$


As can be seen in Table 3, the heat-resistance index was improved from 157.67 (for PSA) to 202.29 (for Si- $\mu$-AlN/PSA). The heat-resistance indexes of $\mu$-AlN/n-AlN/PSA and Si- $\mu$-AlN/n-AlN/PSA are very close to each other, while $\mathrm{Si}-\mu$-AIN/PSA exhibited the highest heat-resistance index. This could be explained as, after surface-treatment of AlN particles, the functional groups attached to the surface of the particles react with the chemical groups of the polymer matrix and create a continuous network reducing chain movements during thermal treatment [22]. Hence, the Si- $\mu$-AlN/PSA becomes thermally more stable. On the other hand, the interfacial interactions were comparatively weak in $\mu$-AlN/n-AlN/PSA due to the non-treated AlN particles resulting in low thermal stability. Similarly, the presence of non-treated $n-A l N$ in Si- $\mu$-AlN/n-AlN/PSA affected the thermal stability negatively in comparison to the $\mathrm{Si}-\mu-\mathrm{AlN} / \mathrm{PSA}$.

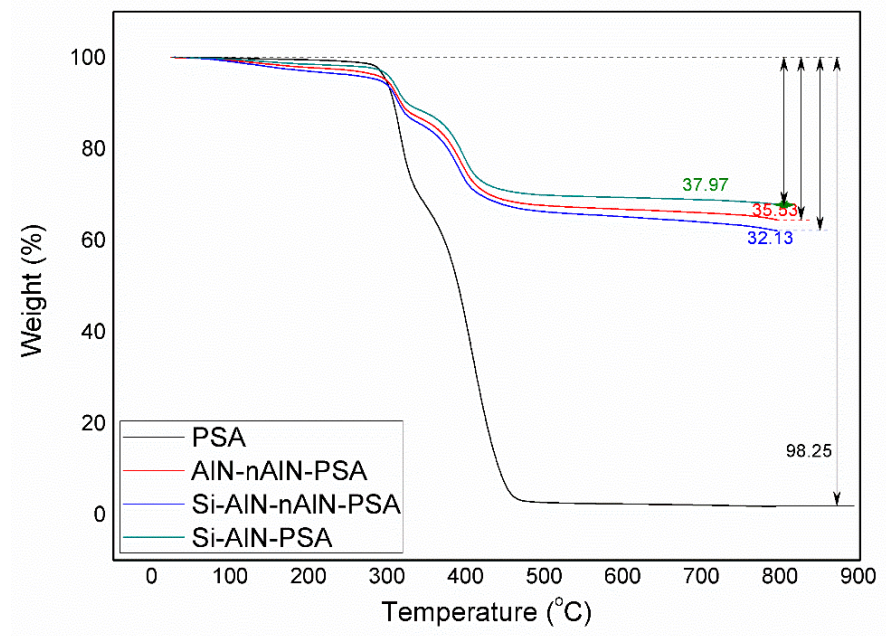

Figure 5. TGA thermograms of PSA, $\mu$-AIN/n-AIN/PSA, Si- $\mu-A I N / n-A I N / P S A$, and Si- $\mu$-AlN/PSA, respectively.

Table 2. Thermogravimetric analysis data of PSA, $\mu-A I N / n-A l N / P S A, ~ S i-\mu-A l N / n-A l N / P S A$, and Si- $\mu$-AlN/PSA.

\begin{tabular}{ccccc}
\hline \multirow{2}{*}{ No. } & Sample Name & \multicolumn{3}{c}{ Weight Loss (\%) } \\
\cline { 3 - 5 } & & I & II & III \\
\hline 1. & PSA & - & 33.97 & 98.25 \\
2. & $\mu-A l N / n-A l N / P S A$ & 3.25 & 15.12 & 35.53 \\
3. & Si- $\mu$-AlN/n-AlN/PSA & 4.56 & 15.83 & 37.97 \\
4. & Si- $\mu$-AlN/PSA & 2.162 & 13.48 & 32.13 \\
\hline
\end{tabular}

Table 3. Heat resistance index of PSA, $\mu-A l N / n-A l N / P S A, S i-\mu-A l N / n-A l N / P S A$, and Si- $\mu-A l N / P S A$.

\begin{tabular}{ccccc}
\hline \multirow{2}{*}{ No. } & Sample Name & \multicolumn{2}{c}{ Weight Loss $\mathbf{( \% )}$} & \multirow{2}{*}{ Heat-Resistance Index $\left({ }^{\circ} \mathbf{C}\right)$} \\
\cline { 3 - 4 } & & $\boldsymbol{T}_{\mathbf{5}}$ & $\boldsymbol{T}_{\mathbf{3 0}}$ & \\
\hline 1. & PSA & 295.80 & 339.10 & 157.67 \\
2. & $\mu$-AlN/n-AlN/PSA & 297.24 & 428.21 & 184.15 \\
3. & Si- $\mu$-AlN/n-AlN/PSA & 287.19 & 415.83 & 178.54 \\
4. & Si- $\mu$-AlN/PSA & 304.41 & 485.14 & 202.29 \\
\hline
\end{tabular}

Figure 6 shows the out-of-the-plane thermal conductivity measured through the Hot Disk method containing the Kapton sensor for all the analyzed samples. As can be seen from the graphs, the thermal conductivity of the neat PSA sample was too low $\left(0.18 \mathrm{Wm}^{-1} \mathrm{~K}^{-1}\right)$. While, after the incorporation of AlN particles, all the samples show improved thermal conductivity. The thermal conductivity for $\mu$-AlN/n-AlN/PSA, Si- $\mu$-AlN/n-AlN/PSA, and Si- $\mu$-AlN/PSA were $0.58,0.68$, and $0.75 \mathrm{Wm}^{-1} \mathrm{~K}^{-1}$, 
respectively, which were $222 \%, 277 \%$, and $316 \%$ higher than that of neat PSA samples. The reason behind this improvement is the formation of a proper thermally conductive network of AlN in the PSA matrix. Generally, neat polymers are thermal insulators. In order to increase their thermal properties, thermally conductive fillers are mixed [23]. When these fillers are dispersed into a higher weight percentage, they form a proper thermally conductive network that helps in phonon transfer during heat treatment [24]. Ironically, the intrinsic value of the filler material can never be attained due to the phonon scattering occurring at the interfacial surface between the filler and matrix. In addition to the weight percentage, size, dispersion, aspect ratio, and alignment of filler material also affect the thermal conductivity of the material [12]. In this study, in the case of $\mu$-AlN/n-AlN/PSA and $\mathrm{Si}-\mu-\mathrm{AlN} / \mathrm{n}$-AlN/PSA, along with the micron-sized AlN particles, nano-sized AlN particles are also mixed. When $\mu$-AlN is dispersed into the matrix, some voids are also generating among them. These n-AlN particles fill these gaps by maintaining a proper thermally conductive network, and, consequently, thermal conductivity increases [25]. In Si- $\mu$-AlN/n-AlN/PSA, silanized $\mu$-AlN was the main reason behind the increased thermal conductivity. The functional groups present on the Si- $\mu$-AlN surface form bonds with chemical groups of PSA, which improves the interactions between AIN particles and the matrix. Hence, Si- $\mu$-AlN/n-AlN/PSA shows a $17 \%$ increase in thermal conductivity as compared to the $\mu$-AlN/n-AlN/PSA. Si- $\mu$-AlN/PSA exhibits the highest thermal conductivity because improved interactions between $\mathrm{Si}-\mu$-AlN particles and the matrix effectively disperse the particles throughout the matrix, which forms an interconnected touching network of Si- $\mu$-AlN particles creating an optimal thermal path through the matrix [26]. The thermal conductivity of Si- $\mu$-AlN/PSA is $10 \%$ more than that of Si- $\mu$-AlN/n-AIN/PSA. The probable reason behind this could be the lack of interactions between non-treated nano-sized AlN particles and matrix/Si- $\mu$-AlN. Moreover, there are reports that say the filler material with smaller size scatters more phonons because of the higher density of interfaces acting as Kapitza resistance leading to lower conductivity (Figure 7) [27]. It can be assumed that the thermal conductivity is more influenced by interactions between filler particles than size. Hence, Si- $\mu$-AlN/n-AlN/PSA has comparatively lower thermal conductivity than the Si- $\mu$-AIN/PSA.
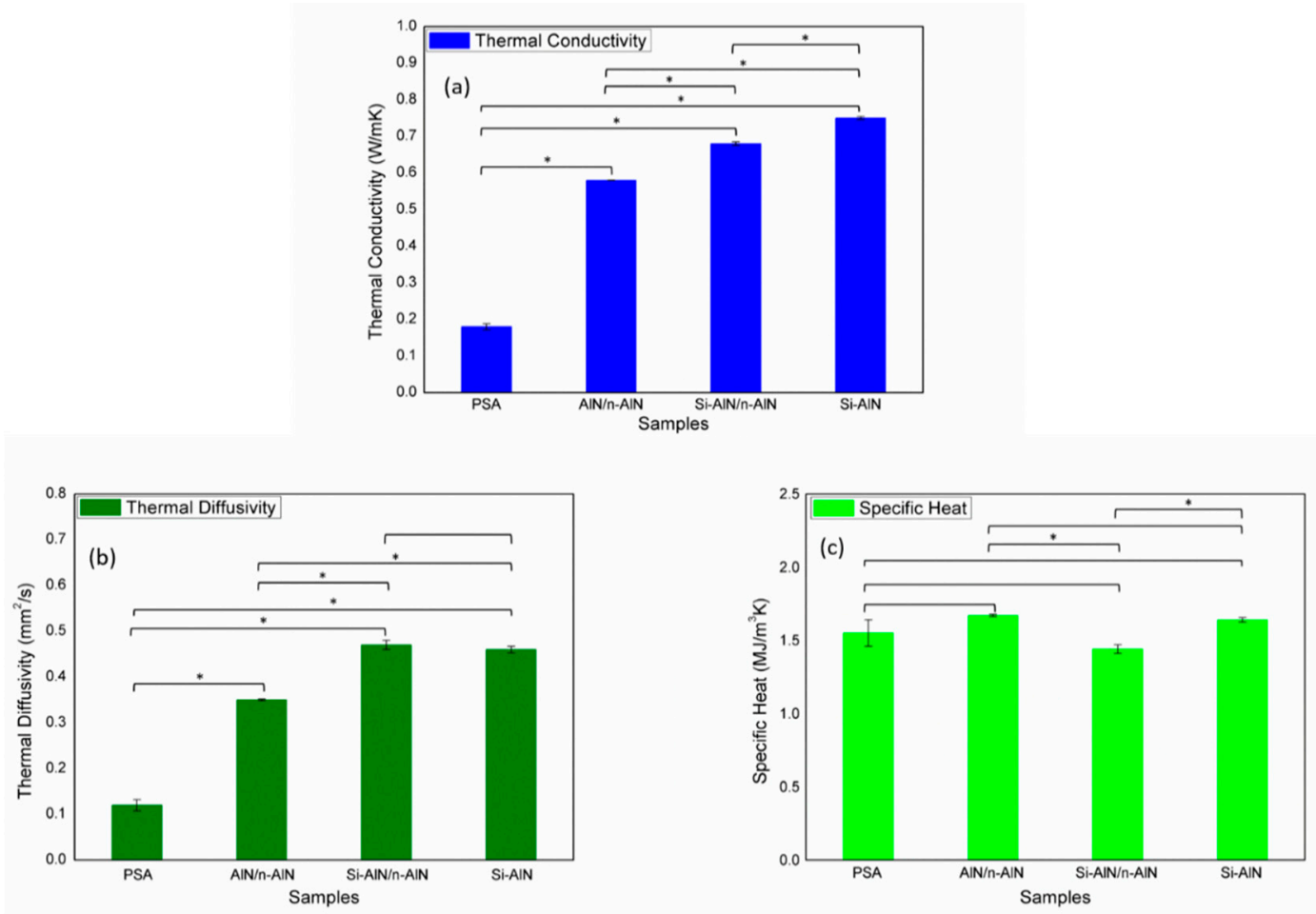

Figure 6. (a) Thermal conductivity, (b) thermal diffusivity, and (c) specific heat of $\mu$-AlN/n-AlN/PSA, $\mathrm{Si}-\mu$-AlN/n-AlN/PSA, and Si- $\mu$-AlN/PSA, respectively (where * represents the p-value $<0.05$, i.e., significant value). 


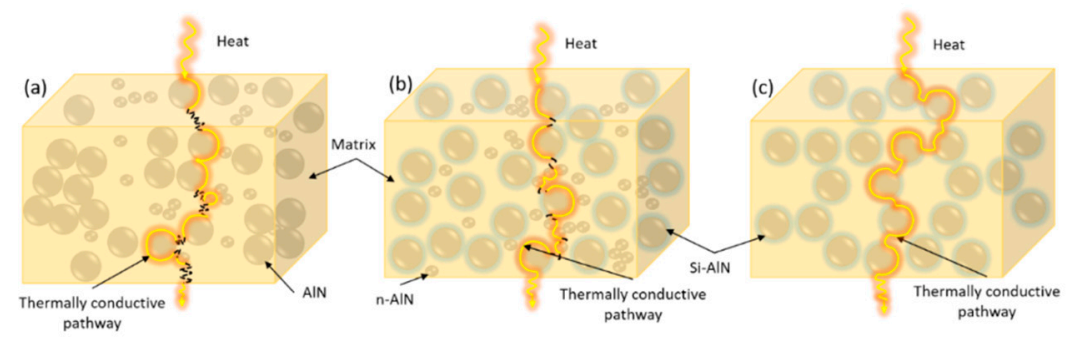

(d)

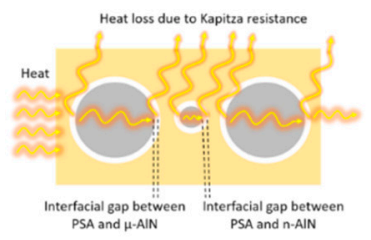

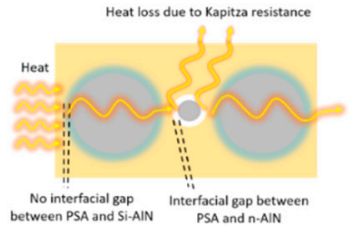

(f)

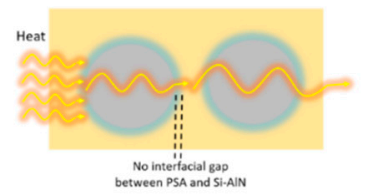

Figure 7. Schematic representation of the heat conduction path in different PSA composites:

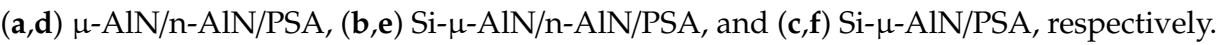

The thermal diffusivity and specific heat capacity of all samples are also represented in Figure 6. As expected, the neat PSA sample shows the lowest thermal diffusivity and specific heat capacity in comparison to the other PSA composites. Among all PSA composites, Si- $\mu-A l N / n-A l N / P S A$ and $\mathrm{Si}-\mu$-AlN/PSA exhibit similar thermal diffusivity, which was higher than that of the $\mu$-AlN/n-AlN/PSA sample. This happens because a continuous phase is created where the polymer matrix is immobilized on the surface of AlN particles (Figure 7) [28]. The order of specific heat capacity for PSA composites was Si- $\mu$-AlN/n-AlN/PSA $\left(1.442 \mathrm{MJ} / \mathrm{m}^{3} \mathrm{~K}\right)<$ PSA $\left(1.549 \mathrm{MJ} / \mathrm{m}^{3} \mathrm{~K}\right)<\operatorname{Si}-\mu$-AlN/PSA $\left(1.639 \mathrm{MJ} / \mathrm{m}^{3} \mathrm{~K}\right)<$ $\mu$-AlN/n-AlN/PSA $\left(1.666 \mathrm{MJ} / \mathrm{m}^{3} \mathrm{~K}\right)$. This could be explained by the improved interfacial interactions between AlN particles and the matrix. As mentioned previously, due to the silane surface-treatment of AlN particles, their dispersion as well as interfacial interaction with the matrix also improved. Hence, a continuous phase is created. Consequently, the density of all the samples differs as samples with treated AlN particles are likely to be denser due to the absence (or a lesser number) of voids and better interactions [29]. In addition, the sample containing the n-AlN could be denser due to the presence of n-AlN particles that fill the void present in the matrix, which leads toward the lower specific heat. Furthermore, in order to analyze the statistical difference of the means (for each cases) of thermal conductivity, thermal diffusivity, and specific heat, ANOVA was performed, and the significance is indicated in Figure 6.

\section{Conclusions}

In this study, the acrylic pressure-sensitive adhesive was prepared by mixing aluminum nitride particles with different sizes. To further improve the interactions between the polymer matrix and AlN particles, particles were treated with 3-APTES, which is a silane coupling agent. The presence of 3-APTES related peaks in FTIR spectra confirmed the successful attachment of chemical moieties on AIN particles. Additionally, SEM images displayed the changes in surface morphology occurred after surface treatment. Thermal conductivity data showed that improving the interfacial interaction between the acrylic matrix and AlN using a surface coupling agent exhibited better thermal conductivity than that of altering the interfacial distance by incorporating n-AlN. This happened because, after treatment, acrylic chains were more easily anchored on the surface of AlN reducing the interfacial thermal resistance while, in another case, the interfacial gap between treated AlN and non-treated $\mathrm{n}$-AlN was responsible for more heat loss.

Author Contributions: Conceptualization, S.J.P. and K.Y.R. Investigation, G.M. Supervision, K.Y.R. Writing-Original Draft, G.M. Writing-Review and Editing, S.J.P. and K.Y.R. All authors have read and agree to the published version of the manuscript. 
Funding: This research received no external funding.

Conflicts of Interest: The authors declare no conflict of interest.

\section{References}

1. Elbreki, A.M.; Alghoul, M.A.; Sopian, K.; Hussein, T. Towards adopting passive heat dissipation approaches for temperature regulation of PV module as a sustainable solution. Renew. Sustain. Energy Rev. 2017, 69, 961-1017. [CrossRef]

2. Otiaba, K.C.; Ekere, N.N.; Bhatti, R.S.; Mallik, S.; Alam, M.O.; Amalu, E.H. Thermal interface materials for automotive electronic control unit: Trends, technology and R\&D challenges. Microelectron. Reliab. 2011, 51, 2031-2043.

3. Ahmadi, Z. Nanostructured epoxy adhesives: A review. Prog. Org. Coat. 2019, 135, 449-453. [CrossRef]

4. Li, Y.; Wong, C.P. Recent advances of conductive adhesives as a lead-free alternative in electronic packaging: Materials, processing, reliability and applications. Mater. Sci. Eng. R Rep. 2006, 51, 1-35. [CrossRef]

5. Dastjerdi, Z.; Cranston, E.D.; Dubé, M.A. Pressure sensitive adhesive property modification using cellulose nanocrystals. Int. J. Adhes. Adhes. 2018, 81, 36-42. [CrossRef]

6. Feldstein, M.M.; Dormidontova, E.E.; Khokhlov, A.R. Pressure sensitive adhesives based on interpolymer complexes. Prog. Polym. Sci. 2015, 42, 79-153. [CrossRef]

7. Park, G.H.; Kim, K.T.; Ahn, Y.T.; Lee, H.; Jeong, H.M. The effects of graphene on the properties of acrylic pressure-sensitive adhesive. J. Ind. Eng. Chem. 2014, 20, 4108-4111. [CrossRef]

8. Guerra, V.; Wan, C.; McNally, T. Thermal conductivity of 2D nano-structured boron nitride (BN) and its composites with polymers. Prog. Mater. Sci. 2019, 100, 170-186. [CrossRef]

9. Mittal, G.; Rhee, K.Y.; Mišković-Stanković, V.; Hui, D. Reinforcements in multi-scale polymer composites: Processing, properties, and applications. Compos. Part B Eng. 2018, 138, 122-139. [CrossRef]

10. Burger, N.; Laachachi, A.; Ferriol, M.; Lutz, M.; Toniazzo, V.; Ruch, D. Review of thermal conductivity in composites: Mechanisms, parameters and theory. Prog. Polym. Sci. 2016, 61, 1-28. [CrossRef]

11. Fu, H.; Wang, Y.; Chen, W.; Zhou, W.; Xiao, J. A novel silanized CoFe2O4/fluorinated waterborne polyurethane pressure sensitive adhesive. Appl. Surf. Sci. 2015, 351, 1204-1212. [CrossRef]

12. Chen, H.; Ginzburg, V.V.; Yang, J.; Yang, Y.; Liu, W.; Huang, Y.; Du, L.; Chen, B. Thermal conductivity of polymer-based composites: Fundamentals and applications. Prog. Polym. Sci. 2016, 59, 41-85. [CrossRef]

13. Tessema, A.; Zhao, D.; Moll, J.; Xu, S.; Yang, R.; Li, C.; Kumar, S.K.; Kidane, A. Effect of filler loading, geometry, dispersion and temperature on thermal conductivity of polymer nanocomposites. Polym. Test. 2017, 57, 101-106. [CrossRef]

14. Li, H.; Chen, W.; Xu, J.; Li, J.; Gan, L.; Chu, X.; Yao, Y.; He, Y.; Li, B.; Kang, F.; et al. Enhanced thermal conductivity by combined fillers in polymer composites. Thermochim. Acta 2019,676, 198-204. [CrossRef]

15. International Organization for Standardization. Plastics-Determination of Thermal Conductivity and Thermal Diffusivity—Part 2: Transient Plane Heat Source (hot disc) Method. (ISO standard no. ISO 22007-2). 2008. Available online: http://www.eyoungindustry.com/uploadfile/file/20151015/20151015144646_47745.pdf (accessed on 18 February 2020).

16. Zhang, Y.; Binner, J. Hydrolysis process of a surface treated aluminum nitride powder-A FTIR study. J. Mater. Sci. Lett. 2002, 21, 803-805. [CrossRef]

17. Lule, Z.; Kim, J. Surface Modification of Aluminum Nitride to Fabricate Thermally Conductive poly (Butylene Succinate) Nanocomposite. Polymers 2019, 11, 148. [CrossRef]

18. Wu, S.-Y.; Huang, Y.-L.; Ma, C.-C.M.; Yuen, S.-M.; Teng, C.-C.; Yang, S.-Y. Mechanical, thermal and electrical properties of aluminum nitride/polyetherimide composites. Compos. Part A Appl. Sci. Manuf. 2011, 42, 1573-1583. [CrossRef]

19. Mittal, G.; Rhee, K.Y.; Park, S.J. Processing and characterization of PMMA/PI composites reinforced with surface functionalized hexagonal boron nitride. Appl. Surf. Sci. 2017, 415, 49-54. [CrossRef]

20. Mittal, G.; Rhee, K.; Park, S. The Effects of Cryomilling CNTs on the Thermal and Electrical Properties of CNT/PMMA Composites. Polymers 2016, 8, 169. [CrossRef]

21. Gu, J.; Du, J.; Dang, J.; Geng, W.; Hu, S.; Zhang, Q. Thermal conductivities, mechanical and thermal properties of graphite nanoplatelets/polyphenylene sulfide composites. RSC Adv. 2014, 4, 22101-22105. [CrossRef] 
22. Kim, M.; Park, S.; Park, J. Effect of the Grafting Reaction of Aluminum Nitride on the Multi-Walled Carbon Nanotubes on the Thermal Properties of the Poly(phenylene sulfide) Composites. Polymers 2017, $9,452$. [CrossRef] [PubMed]

23. Chiu, H.T.; Sukachonmakul, T.; Kuo, M.T.; Wang, Y.H.; Wattanakul, K. Surface modification of aluminum nitride by polysilazane and its polymer-derived amorphous silicon oxycarbide ceramic for the enhancement of thermal conductivity in silicone rubber composite. Appl. Surf. Sci. 2014, 292, 928-936. [CrossRef]

24. Xu, C.; Miao, M.; Jiang, X.; Wang, X. Thermal conductive composites reinforced via advanced boron nitride nanomaterials. Compos. Commun. 2018, 10, 103-109. [CrossRef]

25. Pan, C.; Kou, K.; Zhang, Y.; Li, Z.; Wu, G. Enhanced through-plane thermal conductivity of PTFE composites with hybrid fillers of hexagonal boron nitride platelets and aluminum nitride particles. Compos. Part B Eng. 2018, 153, 1-8. [CrossRef]

26. Pan, C.; Kou, K.; Jia, Q.; Zhang, Y.; Wu, G.; Ji, T. Improved thermal conductivity and dielectric properties of hBN/PTFE composites via surface treatment by silane coupling agent. Compos. Part B Eng. 2017, 111, 83-90. [CrossRef]

27. Wu, H.; Drzal, L.T. High thermally conductive graphite nanoplatelet/polyetherimide composite by precoating: Effect of percolation and particle size. Polym. Compos. 2013, 34, 2148-2153. [CrossRef]

28. Gulotty, R.; Castellino, M.; Jagdale, P.; Tagliaferro, A.; Balandin, A.A. Effects of Functionalization on Thermal Properties of Single-Wall and Multi-Wall Carbon Nanotube-Polymer Nanocomposites. ACS Nano 2013, 7, 5114-5121. [CrossRef]

29. Mittal, G.; Dhand, V.; Ryu, J.; Ryu, J.I.; Rhee, K.Y.; Kim, H.J.; Jung, D.H. Fabrication of Modified MMT/Glass/Vinylester Multiscale Composites and Their Mechanical Properties. J. Nanomater. 2015, 2015, 1-9. [CrossRef]

(C) 2020 by the authors. Licensee MDPI, Basel, Switzerland. This article is an open access article distributed under the terms and conditions of the Creative Commons Attribution (CC BY) license (http://creativecommons.org/licenses/by/4.0/). 